\title{
AN AUDIT OF INDICATIONS AND COMPLICATIONS OF PERCUTANEOUS ENDOSCOPIC GASTROSTOMY
}

\author{
M. Sadik Memon, Asif A. Burney, Javed I. Memon, Waseem Jafri, \\ Saeed Hameed and Khalid Mumtaz
}

\begin{abstract}
OBJECTIVE: To evaluate the indications and complication rate in fifty consecutive patients in whom percutaneous endoscopic gastrostomy (PEG) was attempted.

DESIGN: A descriptive study.

METHODS: Fifty consecutive adults referred to our unit for consideration for primary percutaneous endoscopic gastrostomy feeding between November 1999 and October 2001 at The Aga Khan University Hospital, Karachi and Isra University Hospital, Hyderabad were evaluated prospectively.

RESULTS: PEG insertions were successful in all fifty patients (29 Males, 21 Females). The indications for PEG were neurogenic (80\%), recurrent aspiration, not the gastric contents (8\%), head and neck tumors (6\%), esophageal tumor (4\%) and dermatomyositis (2\%). Ninety six percent of the patients had the procedure as inpatients while $4 \%$ were outpatients when the procedure was performed. Eighty percent of the patients had at least one follow-up after the tube insertion. Major complications were peritonitis $(2 \%)$ and aspiration of gastric contents (4\%). Minor complications were PEG site infection (16\%), accidental tube removal (10\%), PEG migration (6\%) and PEG blockage (4\%). One patient died due to peritonitis. Oral feed was resumed in $12 \%$ of the patients and the tube was removed subsequently.

CONCLUSION: PEG is a feasible and safe procedure in our set up. It provides adequate feeding support and has low mortality rate even in patients, debilitated secondary to their underlying disease.
\end{abstract}

KEY WORDS: Gastrostomy. Indication. Complication. Peritonitis. Mortality.

\section{INTRODUCTION}

Percutaneous endoscopic gastrostomy (PEG) is a relatively new method and has become a mainstay of nutritional support for individuals with swallowing dysfunction and inadequate caloric intake who have a functionally intact gastrointestinal mucosa. Ponsky and Gauderer introduced the procedure in $1980^{1}$. It allows permanent enteral access without the need of laparotomy and general anesthesia. Indications of PEG are the same as for traditional gastrostomy, and include inability to swallow due to neurological impairment, oropharyngeal neoplasm and fascial trauma. Though PEG insertion is simple, but the endoscopist must be aware of its associated serious and potentially lethal complications. Furthermore, patient selection and thorough attention to details are essential for safe PEG placement. Over the past few years, a rapidly growing number of referral was made to the Gastroenterology section for PEG insertion. This paper describes the results of PEG insertion in 50 consecutive patients. Our objective was to determine feasibility, indications, procedure related complications and mortality and to follow, where possible, patients progress.

\section{PATIENTS AND METHODS}

We studied 50 consecutive adult patients prospectively, referred for PEG placement during the period of November 1999 to October 2001 at The Aga Khan University Hospital Karachi and Isra University Hospital Hyderabad, Sindh. All gastrostomies were performed in endoscopy suite. Parenteral antibiotic (one-gram intravenous cefotaxime) was administered to all patients for prophylaxis. Midazolam was used for conscious sedation and topical pharyngeal spray of $4 \%$ xylocaine solution was given before the insertion of the gastroscope. All patients were given supplementary intranasal oxygen and oxygen saturation and pulse rate were monitored with pulse oxymeter. Esophagogastroduodenoscopy was done prior to PEG insertion in all patients and a standard 24 French (WILSON COOK) PEG tube was placed using "PUSH TECHNIQUE". Patients were not fed through the PEG tube for 12 to 18 hours, after placement of PEG. Data were analyzed on Statistical 
Program for Social Sciences (SPSS) version 10.

\section{RESULTS}

PEG tube was placed into 50 consecutive patients, comprising of 21 females and 29 males. Their ages ranged from 24 to 90 years. Two third of the patients were more than 55 years old as shown in figure I. The most common indication for PEG tube placement was neurological disorders (80\%). These included Cerebrovascular accident $(70 \%)$, motor neuron disease $(2 \%)$, Tuberculoma of brain $(2 \%)$, Parkinson's disease (2\%), brain tumor $(2 \%)$ and encephalitis $(2 \%)$. This was followed by oropharyngeal obstruction and general debility with difficulty in swallowing (20\%) as shown in figure II.Gastrostomy tube was successively placed in all 50 patients without complications during the procedure.Major complications included aspiration pneumonia in two patients (treated with antibiotics successfully) and peritonitis in one patient. The patient who developed peritonitis was an 84 years old lady with multi infracts dementia and metastatic cervical cancer. She was treated conservatively but she developed septic shock and died. The overall procedure related minor complications rate was $42 \%$. The most common minor complication was PEG site (wound) infection observed in $16 \%$ of patients and in each it resolved with enteral (via PEG tube) or parenteral antibiotics. The PEG tube had to be removed in three patients due to wound infection (Figure III). Other minor complications included an accidental PEG tube removal $(n=5$, in each patient the PEG tube was replaced by Foleys catheter through the sinus tract), PEG displacement $(n=3)$, PEG stoma leak ( $n=3$, treated with stoma adhesive) and PEG block $(n=1$, failed to open it and subsequently replaced by Foleys catheter).

\section{FIGURE I: AGE DISTRIBUTION OF CASES}

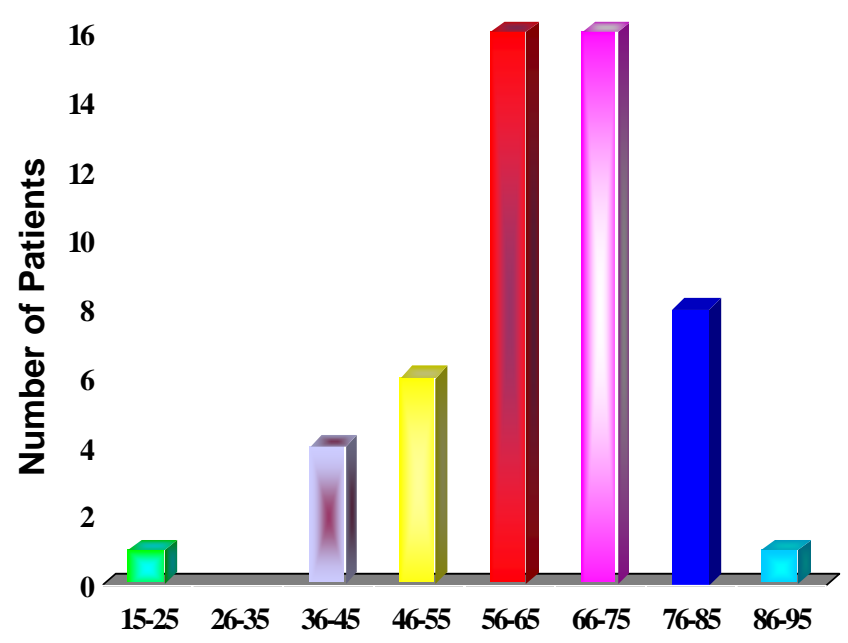

\section{IN YEARS $(n=50)$ FIGURE II: INDICATIONS}

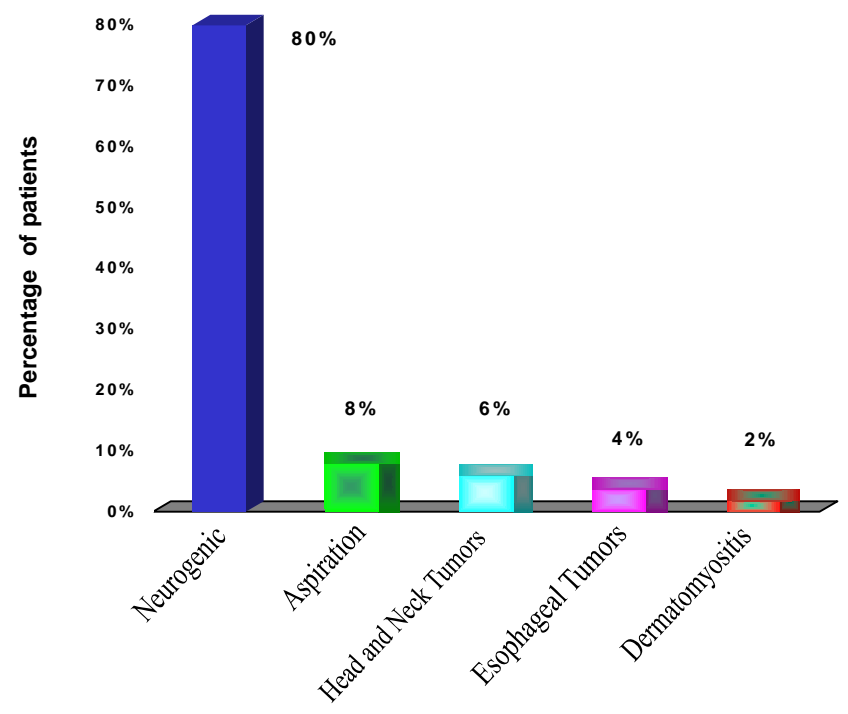

FIGURE III: COMPLICATIONS ASSOCIATED WITH PEG $(n=50)$

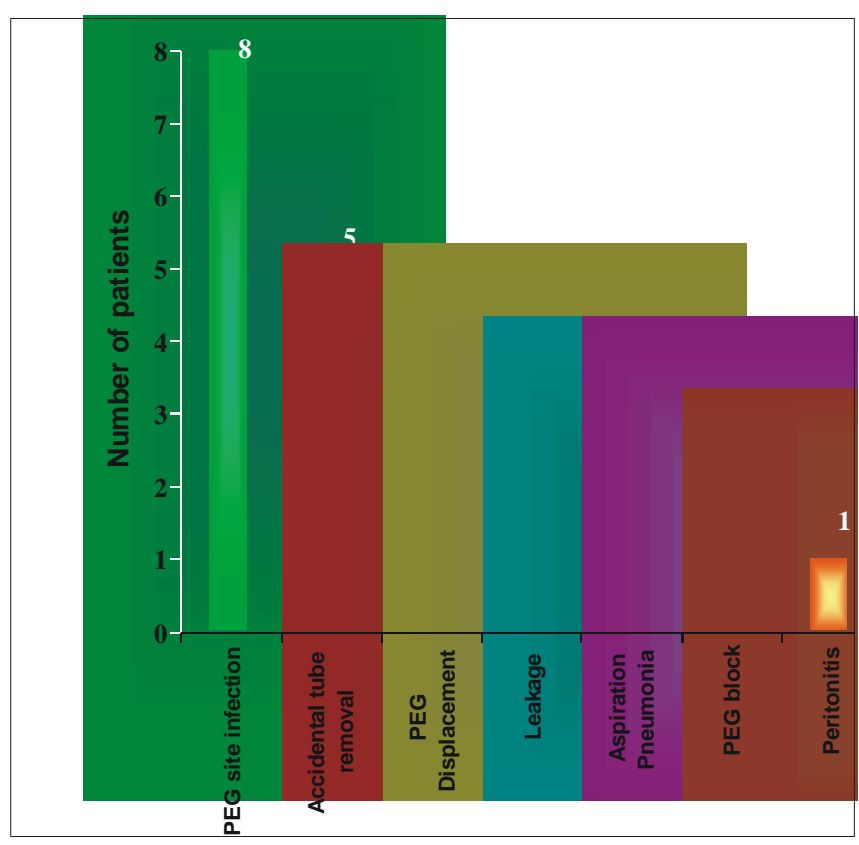

IN PEG CASES $(n=50)$

\section{DISCUSSION}

This study has observed $100 \%$ success rate for PEG insertion via push technique. This is in agreement with several other reports in which the failure rate was less than $10 \%{ }^{2}$. We did not experience any immediate procedure related complication or mortality, despite many of the patients being frail and debilitated. This study also shows that the major 
indication for PEG insertion was a neurological disorder, with the majority of patients suffering from cerebrovascular accidents. Neurological disorders have been reported as the leading indication in other series $^{3}$. Other indications in this study were same as have been reported in literature, that is oropharyngeal and esophageal obstruction ${ }^{4}$.

This study highlights the importance of initial decision to place a PEG for enteral feeding in patients with multiple or serious medical problems. A PEG insertion should be considered for long term enteral feeding in patients who are unable to maintain adequate nutrition by mouth with otherwise functioning gut mucosa. For this study, in long term follow up (6 months) twelve (24\%) patients were continued on PEG feeding maintaining their weight. Other six (12\%) patients were recovered and able to take orally and the PEG tube was pulled out permanently. Rest of the patients $(62 \%)$ had been lost to follow up beyond 6 months. Further in long term feeding, PEG insertion appears to provide improved patient and caregiver's confidence and acceptance, while having the advantage of a lower risk of aspiration and being cosmetically less disfiguring as compared to a nasogastric tube.

Co-morbid medical conditions are vital in determining the suitability and timing of a PEG insertion. An absolute contraindication to PEG placement is the inability to bring the anterior gastric wall in apposition to the anterior abdominal wall ${ }^{2}$. Gastric resection, ascites, huge hepatomegaly, and obesity are some conditions, which may impede gastric transillumination and subsequent PEG placement. In our case series, none of the contra-indications were encountered. This probably reflects better case selection due to knowledge and awareness on the part of the referring teams. Antibiotic prophylaxis is routinely advised. It significantly reduces the percentage of PEG site infection. In one study it has reduced the rate from $30 \%$ to $7 \%{ }^{5}$. The choice of antibiotics is not clear, with many studies using a variety of regimen ${ }^{6,7}$. Most of our patients were already on broad-spectrum antibiotics due to other reasons such as, hypostatic pneumonia, central line placements and urinary catheterization. Three retrospective studies have demonstrated reduced mortality and morbidity with endoscopic insertion of gastrostomy compared with surgical insertion under general anesthesia ${ }^{8,9}$. Complications of PEG are infrequent, with a procedure related mortality rate of $1-2 \%$ and morbidity of 3-12 \% in the largest reported series $^{2,4}$. Our results are consistent with these published results. For example, the mortality and morbidity of our patients is comparable to what has been reported by Hull et $\mathrm{al}^{4}$. Only 6 of 50 patients
(12\%) had their PEG removed because they had regained the ability to maintain their nutrition by oral feeding. The majority of patients (those in follow up) at the end of this review still had their PEG in situ and it was functioning. This suggests that for the majority of patients, PEG was placed during the terminal stage of their disease, with little chance of spontaneous or treatment related recovery. This further highlights the feasibility of placing PEG even in terminally ill and debilitated patients to improve the quality of life.

There are certain limitations of the study. Firstly, longterm follow up is not available on all patients. Secondly, survival data are not available, again owing largely due to the lack of follow up. Thirdly, because of the relatively few complications, it was not possible to identify disease or patient related factors predicting complications with PEG placement.

\section{CONCLUSION}

In our experience, the PEG is an effective means of feeding when oral feeding is not possible despite a functioning gastrointestinal mucosa. The PEG placement is safe, acceptable and easily tolerable even in debilitated patients. Candidates for PEG feeding should need enteral nutrition for more than four to six weeks and have a prognosis that justifies nutritional support.

\section{REFERENCES}

1. Gauderer MWL, Ponsky JL, Izant RJ Jr. Gastrostomy without laparotomy: a percutaneous endoscopic technique. J Pediatr Surg 1980. 15: $872-5$.

2. Larson DE, Burton DD, Ott BJ et al. Percutaneous endoscopic gastrostomy: indications, success, complications and mortality in 314 consecutive patients. Gastroenterology 1987; 93: 48 - 52.

3. Nicholson FB, Korman MG, Richardson MA. Percutaneous endoscopic gastrostomy: a review of indications, complications and outcome 2000:15; $21-25$.

4. Hull MA, Rawlings J, Murray $F$ et al. Audit of long-term enteral nutrition by percutaneous endoscopic gastrostomy. Lancet 1993; 341: 869- 72 .

5. Jain NK, Larson DE, Schroeder KW et al. Antibiotic prophylaxis for percutaneous endoscopic gastrostomy: a prospective, randomized controlled clinical trail. Ann Intern Med 1987; 107: 824 - 8.

6. Sturgis TM, Yancy W, Cole JC et al. Antibiotic prophylaxis in percutaneous endoscopic gastrostomy. Am J Gasroenterol 1996; 91: 
3201-4.

7. Rey JR, Axon A, Budzynska A et al. Antibiotic prophylaxis for gastrointestinal endoscopy. guidelines of the European Society of Gastrointestinal Endoscopy (ESGE). Endoscopy
1998; 30: $318-24$.

8. Grant JP. Comparison of percutaneous endoscopic gastrostomy with Stamm gastrostomy. Ann J Surg 1988; 207: 598 - 603.

AUTHOR AFFILIATION:

Dr. M. Sadik Memon (Corresponding Author)

Associate Professor

Department of Medicine, Isra University Hospital,

P.O.Box \# 313, Hala Road Hyderabad 71000, Sindh, Pakistan.

Email: sadik369@yahoo.com

Dr. Asif A. Burney

Assistant Professor

Department of Medicine, Isra University Hospital,

P.O.Box \# 313, Hala Road Hyderabad 71000, Sindh, Pakistan.

Dr. Javed I. Memon

Associate Professor

Department of Medicine, Isra University Hospital,

P.O.Box \# 313, Hala Road Hyderabad 71000, Sindh, Pakistan.

Dr. Waseem Jafri

Professor of Medicine

Aga Khan University Hospital, Karachi - Pakistan

Dr. Saeed Hameed

Professor of Medicine

Aga Khan University Hospital, Karachi - Pakistan

Dr. Khalid Mumtaz

Senior Instructor, Department of Medicine

Aga Khan University Hospital, Karachi - Pakistan 